\title{
Avaliação do desempenho hemodinâmico do dispositivo de assistência ventricular InCor como substituto do coração esquerdo
}

\author{
Anderson BENÍCIO*, Luiz Felipe P. MOREIRA*, Sérgio HAYASHIDA*, Idagene A. CESTARI*, \\ Adolfo A. LEIRNER* ${ }^{\star}$, Noedir A. G. STOLF* ${ }^{\star}$, Adib D. JATENE*
}

RBCCV 44205-465

Benício A, Moreira L F P, Hayashida S, Cestari I A, Leirner A A, Stolf N A G, Jatene A D - Avaliação do
desempenho hemodinâmico do dispositivo de assistência ventricular InCor como substituto do coração
esquerdo. Rev Bras Cir Cardiovasc 1999; 14 (3): 237-46

RESUMO: Fundamento: A assistência circulatória mecânica é uma opção terapêutica em casos de choque cardiogênio refratário ao tratamento farmacológico, sendo freqüentemente utilizada como ponte para o transplante cardíaco.

Objetivo: Avaliar o desempenho do Dispositivo de Assistência Ventricular (DAV) desenvolvido pela Bioengenharia do Instituto do Coração, quando implantado com substituto do coração esquerdo.

Casuística e Métodos: Foram estudados 10 bezerros da raça Girolando com peso médio de $73 \mathrm{~kg}$. $O$ implante DAV-InCor foi realizado com a cânula de drenagem posicionada no átrio esquerdo (AE) ou na ponta do ventrículo esquerdo (VE) e a cânula de reposição implantada na aorta torácica descendente. As pressões do coração direito e esquerdo, débito cardíaco e o fluxo do DAV foram determinados antes e após a indução farmacológica de insuficiência miocárdica, na vigência de diferentes níveis de vácuo do sistema de drenagem.

Resultados: Com a drenagem no AE, os valores do fluxo do DAV foram de 2,2 $\pm 0,5 \mathrm{l} / \mathrm{min}$ na condição sem vácuo, $3,7 \pm 0,4 \mathrm{l} / \mathrm{min}$ com vácuo de $10 \mathrm{mmHg}$, de 4,3 $\pm 0,4 \mathrm{com}$ vácuo de $20 \mathrm{mmHg}$ e de 4,8 $\pm 0,6 \mathrm{com}$ vácuo de $30 \mathrm{mmHg}$. Os valores da pressão de $A E$ foram de 11,7 $\pm 6 ; 9,8 \pm 5,3,8,5 \pm 4,4$ e 5,6 $\pm 3,3 \mathrm{mmHg}$ nas mesmas condições, respectivamente. Com a cânula ventricular, o fluxo DAV foi de $4,2 \pm 0,6$ na condição sem vácuo e de $4,4 \pm 0,7$ com vácuo de $10 \mathrm{mmHg}$, sendo observados valores de pressão de $A E$ de $11,1 \pm 2$ e 10,3 $\pm 3,5 \mathrm{mmHg}$ nas duas condições. Esses resultados foram observados em condições hemodinâmicas semelhantes, sendo o fluxo do DAV responsável por um porcentual maior do débito cardíaco total conforme o nível de vácuo. Esse porcentual foi de $86 \pm 13 \%$ com a cânula atrial e vácuo de $30 \mathrm{mmHg}$ e de $97 \pm 3 \%$ com a drenagem ventricular e vácuo de $10 \mathrm{mmHg}$.

Conclusões: O DAV-InCor mostrou-se eficiente como substituto do ventrículo esquerdo. O desempenho deste dispositivo foi melhor quanto maior o nível de vácuo no sistema de drenagem e com a utilização de cânula ventricular.

DESCRITORES: Ventrículo cardíaco, cirurgia. Coração auxiliar.

Trabalho realizado no Instituto de Coração do Hospital das Clínicas da Faculdade de Medicina da Universidade de São Paulo. São Paulo, SP, Brasil. Apresentado ao 26을 Congresso Nacional de Cirurgia Cardíaca. Fortaleza, CE, 8 a 10 de abril, 1999.

* Do Instituto do Coração do Hospital das Clínicas da Faculdade de Medicina da Universidade de São Paulo.

Endereço para correspondência: Anderson Benício. Av. Dr. Enéas de Carvalho Aguiar, 44. Cerqueira César. Divisão Cirúrgica. São Paulo, SP, Brasil. CEP 05403-000.e-mail: abenicio@uol.com.br 
Benício A, Moreira L F P, Hayashida S, Cestari I A, Leirner A A, Stolf N A G, Jatene A D - Avaliação do desempenho hemodinâmico do dispositivo de assistência ventricular InCor como substituto do coração esquerdo. Rev Bras Cir Cardiovasc 1999; 14 (3): 237-46.

\section{INTRODUÇÃO}

Nos últimos anos, o transplante cardíaco tem se caracterizado como a terapêutica de escolha no tratamento das doenças cardíacas terminais. Entretanto, o transplante cardíaco apresenta algumas dificuldades em sua aplicabilidade. As contra-indicações médicas e psicossociais, a falta de doadores e o atual caráter inespecífico da imunossupressão são limitações desse procedimento.

Frente a este cenário, surge a necessidade de lançarmos mão de outras formas de tratamento ou mesmo de procedimentos alternativos. Novas técnicas vêm sendo desenvolvidas e aprimoradas no sentido de ampliar a expectativa de vida dos pacientes que aguardam um órgão na fila de transplantes. O tratamento cirúrgico pode ser variável conforme a etiologia da insuficiência cardíaca. Além do transplante cardíaco, a revascularização do miocárdio, a substituição valvar, a cardiomioplastia dinâmica, a ventriculectomia parcial esquerda, o implante de desfibrilador automático e o implante de marcapasso ventricular esquerdo são algumas alternativas.

Entretanto, nem todos pacientes têm a possibilidade de algum tipo de tratamento alternativo que não o transplante. Surge, então, como última perspectiva nos pacientes que evoluem em choque cardiogênico, refratário à terapêutica medicamentosa, à aplicação de dispositivos de assistência circulatória mecânica.

A utilização de dispositivos de assistência circulatória mecânica tem como objetivo converter uma condição terminal em uma condição clínica estável que permita a realização de um transplante cardía$\mathrm{co}$, funcionando assim como ponte para o transplante. Os dispositivos geralmente mais utilizados com este fim são o balão intra-aórtico, as bombas centrífugas, os ventrículos artificiais e o coração artificial total.

Os ventrículos artificiais se apresentam de várias formas. Podem ser implantáveis ou paracorpóreos, com mecanismo de funcionamento pneumático ou eletromecânico. Todos eles apresentam a característica de assistência circulatória pulsátil, o que torna a assistência mais fisiológica, ao contrário das bombas axiais e centrífugas, de característica não pulsátil (1).

O uso dos ventrículos artificiais pode ser instituído como assistência ventricular esquerda, direita ou biventricular. Uma desvantagem dos dispositivos de assistência ventricular de acionamento pneumático é o "drive" de grandes dimensões, gerando desconforto e falta de praticidade no que diz respeito à locomoção do paciente. Porém, já existem modelos eletromecânicos, nos quais o paciente tem a condição de realizar atividades comuns sem grandes restrições.
A indicação principal para o implante de dispositivos de assistência circulatória mecânica é para os pacientes que aguardam um órgão na fila de transplante. Outros grupos que também se encaixam para esta indicação são os pacientes que evoluem com choque cardiogênico pós-infarto do miocárdio, os pacientes que apresentam miocardites com possibilidade de recuperação e os pacientes que apresentam choque cardiogênico no pós-operatório de operações cardíacas ${ }^{(1-3)}$. De modo geral, entre $60 \%$ e $70 \%$ dos pacientes submetidos à assistência circulatória mecânica conseguem ser submetidos a transplante cardíaco, sendo observada mortalidade hospitalar após o transplante em torno de $20 \%$ (1).

O dispositivo de assistência ventricular (DAV)InCor teve seu desenvolvimento no início da década de $90^{(4)}$. A sua primeira aplicação clínica ocorreu em 1993, em paciente que apresentou choque cardiogênico refratário à terapia medicamentosa e submetido a transplante cardíaco após o quarto dia de assistência, recebendo alta hospitalar (5).

Mais recentemente, este dispositivo sofreu alterações estruturais importantes, como a aplicação de vácuo no sistema de drenagem, o que resultou em substancial melhora em seu desempenho ${ }^{(6)}$. Neste trabalho são apresentados os resultados do desempenho hemodinâmico do DAV-InCor quando implantado na assistência ao coração esquerdo com a cânula de drenagem implantada no átrio esquerdo ou na ponta do ventrículo esquerdo.

\section{MATERIAL E MÉTODOS}

Este estudo foi desenvolvido na Divisão de Bioengenharia do Instituto do Coração do Hospital das Clínicas da Faculdade de Medicina da Universidade de São Paulo. O DAV-InCor consiste de duas câmaras confeccionadas de epoxi separadas por uma membrana de poliuretano, formando uma câmara pneumática e outra sangüínea, que é revestida por uma suave camada de poliuretano na região da junção das duas câmaras. A câmara sangüínea apresenta duas aberturas: uma para o fluxo de entrada, na qual se interpõe uma prótese valvar biológica com diâmetro de $25 \mathrm{~mm}$ e outra para o fluxo de saída, onde se interpõe uma prótese valvar biológica com diâmetro de $23 \mathrm{~mm}$. Seu modo de acionamento é pneumático ("fill to empty") comandado por um "drive" de sistema computadorizado. Em comparação ao modelo inicial do DAV-InCor, a inovação presente é a incorporação de um sistema de vácuo no circuito de drenagem do dispositivo. Foram utilizados 10 bezerros da raça Girolando com peso médio de $73 \mathrm{~kg}$. Os animais foram divididos em dois grupos. No Grupo I, 6 animais foram sub- 
Benício A, Moreira L F P, Hayashida S, Cestari I A, Leirner A A, Stolf N A G, Jatene A D - Avaliação do desempenho hemodinâmico do dispositivo de assistência ventricular InCor como substituto do coração esquerdo. Rev Bras Cir Cardiovasc $1999 ; 14$ (3): 237-46.

metidos à assistência ventricular esquerda com a cânula de drenagem colocada no átrio esquerdo, sendo feita a avaliação do desempenho em condição normal e com a indução de insuficiência miocárdica com infusão de betabloqueador (propanolol) na dose de $1 \mathrm{mg} / \mathrm{kg}$ de peso. No Grupo II, foi realizada a assistência ventricular esquerda em 4 animais, com o sistema de drenagem com canulação ventricular, sendo apenas feita a avaliação do desempenho em condição normal.

\section{Procedimento Anestésico}

Os animais receberam como medicação préanestésica cloridrato de tiazina na dose de $0,5 \mathrm{mg} /$ $\mathrm{kg}$. A anestesia foi realizada através da administração de midazolam, cloridrato de fentanila e pancurônico.

A monitorização constitui no registro do eletrocardiograma, da pressão arterial média através da dissecção e cateterização de uma artéria periférica, das pressões hemodinâmicas centrais através da inserção de um cateter tipo Swan-Ganz (Baxter), e da medida da pressão de átrio esquerdo através de um cateter tipo Müller. Foram realizadas medidas de débito cardíaco no transcorrer dos procedimentos, bem como dosagens do hematócrito, hemoglobina e gasometria arterial. Para hidratação, administração de drogas e derivados de sangue, foi utilizada uma veia periférica cateterizada por dissecção.

\section{Procedimento Cirúrgico}

Com o bezerro posicionado em decúbito lateral direito, foi realizada uma toracotomia lateral esquerda no quinto espaço intercostal esquerdo.

\section{Grupo I}

Após a administração de heparina $(2,0 \mathrm{mg} / \mathrm{kg})$, foi inserida uma cânula de drenagem no átrio esquerdo através de sutura em bolsa e outra cânula de infusão na aorta descendente através de sutura término-lateral. O cateter de Müller foi inserido no átrio esquerdo por meio de sutura em bolsa. Conectou-se o dispositivo em suas cânulas com a retirada completa de ar de seu interior.

\section{Grupo II}

Neste grupo, a cânula de drenagem foi inserida no ventrículo esquerdo. Para isto, houve a necessidade da instalação de circulação extracorpórea (CEC) de maneira convencional. Após a administração de heparina $(4,0 \mathrm{mg} / \mathrm{kg})$, uma cânula de drenagem foi inserida no átrio direito através de sutura em bolsa e outra de infusão na aorta descendente também através de sutura em bolsa. A CEC foi então estabelecida, sendo a seguir posicionada a cânula de drenagem na porção apical do ventrículo esquerdo. Esta cânula foi inserida na região apical por meio de pontos separados apoiados em "pledgets". Já a cânula de infusão foi anastomosada na aorta torácica descendente, do mesmo modo que no Grupo I. O cateter de Müller também foi inserido no átrio esquerdo do mesmo modo que no Grupo I. Foi estabelecida então a conexão com o dispositivo, com a completa retirada de ar e o início de seu funcionamento.

\section{Parâmetros Estudados}

\section{Grupo I}

Neste grupo, o experimento foi dividido em duas fases, utilizando 6 animais. A primeira foi realizada em condições normais e a segunda em condições de insuficiência miocárdica, obtida de forma aguda pela administração intravenosa de beta-bloqueador (Propanolol) na dose de 1,0 mg/kg. Nas duas fases, o dispositivo foi acionado inicialmente sem a aplicação de vácuo no sistema de drenagem. Posteriormente, o vácuo foi acionado com a pressão no valor de $10 \mathrm{mmHg}$, a qual foi elevada para $20 \mathrm{mmHg}$ e $30 \mathrm{mmHg}$. Em cada situação, o dispositivo ficou em funcionamento por um período de $10 \mathrm{~min}$ com o objetivo de se obter adequada estabilidade hemodinâmica.

Foram então realizadas as seguintes medidas hemodinâmicas: freqüência cardíaca, pressão arterial sistêmica, pressão de átrio esquerdo, pressão de artéria pulmonar, pressão de átrio direito, débito cardíaco e fluxo do dispositivo.

Com o objetivo de manter as condições gerais do animal, concomitantemente, foram realizadas dosagens de gases arteriais, hematócrito, hemoglobina e potássio. A administração de sangue homólogo foi realizada com o objetivo de manter a volemia e níveis adequados de hemoglobina.

Após a realização do experimento, os animais foram sacrificados com a administração intravenosa de potássio em alta concentração.

\section{Grupo II}

Neste grupo, 4 animais foram estudados somente em condições normais e o experimento foi dividido em duas fases. Inicialmente o DAV-InCor foi 
Benício A, Moreira L F P, Hayashida S, Cestari I A, Leirner A A, Stolf N A G, Jatene A D - Avaliação do desempenho hemodinâmico do dispositivo de assistência ventricular InCor como substituto do coração esquerdo. Rev Bras Cir Cardiovasc 1999; 14 (3): 237-46.

acionado sem a aplicação de vácuo no sistema de drenagem e numa segunda fase, com a aplicação de vácuo no sistema de drenagem com pressão no valor de $10 \mathrm{mmHg}$.

Foram estudados os mesmos parâmetros que no Grupo I, mantendo a mesma metodologia.

Após a realização do experimento, os animais foram sacrificados com a administração intravenosa de potássio em alta concentração.

\section{Análise Estatística}

Foram determinados os valores médios e os respectivos desvios-padrão de cada uma das variáveis. Os comportamentos dos parâmetros analisados com os diferentes níveis de vácuo no sistema de drenagem foram estudados pelo teste de análise de variância. Foram considerados significativos os valores de $p<0,05$.

\section{RESULTADOS}

Dos dez experimentos realizados, em 3 (dois no Grupo I e um no Grupo II) ocorreram problemas técnicos ou instabilidade do animal o que levou a invalidar algumas medidas hemodinâmicas. O problema mais crítico e comum ocorrido durante os experimentos foi o quadro de insuficiência respiratória após a administração de sangue. Apesar da otimização do suporte ventilatório e ajustes de gasometrias, o animal desenvolvia dessaturação arterial com importante repercussão hemodinâmica, o que promoveu a interrupção de alguns experimentos. A administração de sangue se fez necessária sempre que o hemotócrito atingia níveis menores que $30 \%$.
Nos experimentos do Grupo II, 2 animais apresentaram fibrilação ventricular após o início da CEC, necessitando de desfibrilação $(10 \mathrm{~J})$ com boa recuperação em ritmo sinusal.

\section{Grupo I}

$\mathrm{Na}$ Tabela 1 são apresentados os valores médios e o desvio padrão da freqüência cardíaca, das pressões arterial, de átrio esquerdo, de átrio direito e de artéria pulmonar, bem como do débito cardíaco e do fluxo do DAV-InCor, obtidos em condição normal e após a indução de disfunção ventricular pela infusão de betabloqueador. Pode-se observar que não houve variação significativa dos valores da freqüência cardíaca e da pressão arterial nas várias condições estudadas. Por outro lado, foram observados valores mais elevados das pressões de átrio direito, artéria pulmonar e de átrio esquerdo após a indução de disfunção ventricular. Estas alterações ocorreram concomitantemente à redução do débito cardíaco na mesma condição.

Em relação ao comportamento do DAV-InCor, houve elevação progressiva do fluxo do dispositivo com o aumento do nível de vácuo no sistema de drenagem nas duas condições estudadas, como mostra o Gráfico 1. Esta elevação foi responsável por um porcentual progressivamente maior do débito cardíaco total, principalmente na presença de disfunção ventricular (Gráfico 2). Esse porcentual foi em média de $84 \pm 13 \%$ com a cânula atrial e vácuo de $30 \mathrm{mmHg}$, quando analisado na situação normal e de até $88 \pm 10 \%$ nas mesmas condições após a indução de disfunção ventricular.

O Gráfico 3 mostra a relação entre a pressão de átrio esquerdo e o fluxo do dispositivo nas diversas condições estudadas, sendo também apresentadas as curvas de desempenho obtidas através de

TABELA 1

PARÂMETROS HEMODINÂMICOS E DESEMPENHO DO DAV-InCor CÂNULA ATRIAL (GRUPOI)

\begin{tabular}{|c|c|c|c|c|c|c|c|c|}
\hline & & $\begin{array}{l}F C \\
(B P M)\end{array}$ & $\begin{array}{l}A D \\
(M M H G)\end{array}$ & $\begin{array}{l}P A P \\
\text { (MMHG) }\end{array}$ & $\begin{array}{l}A E \\
(M M H G)\end{array}$ & $\begin{array}{l}P A M \\
(M M H G)\end{array}$ & $\begin{array}{l}D C \\
(L / M I N)\end{array}$ & $\begin{array}{l}F x \\
(L / M I N)\end{array}$ \\
\hline \multirow[t]{2}{*}{ V 0} & 1 & $93,5 \pm 35,39$ & $9,58 \pm 3,11$ & $30,25 \pm 8,32$ & $9,55 \pm 4,13$ & $95,67 \pm 27,12$ & $5,75 \pm 1,43$ & $2,92 \pm 0,29$ \\
\hline & 2 & $66,67 \pm 41,03$ & $13,38 \pm 7,51$ & $36,32 \pm 23,28$ & $12,26 \pm 7,86$ & $101,8 \pm 27,45$ & $3,31 \pm 2,00$ & $2,55 \pm 0,60$ \\
\hline \multirow[t]{2}{*}{ V 10} & 1 & $92,0 \pm 38,46$ & $9,58 \pm 2,52$ & $31,75 \pm 7,528$ & $6,6 \pm 2,53$ & $105 \pm 26,41$ & $5,91 \pm 0,81$ & $3,39 \pm 0,65$ \\
\hline & 2 & $59,33 \pm 34,57$ & $13,75 \pm 7,44$ & $37,0 \pm 26,03$ & $9,62 \pm 6,44$ & $107 \pm 19,53$ & $3,61 \pm 1,85$ & $3,85 \pm 0,38$ \\
\hline \multirow[t]{2}{*}{ V 20} & 1 & $86,0 \pm 34,61$ & $10,25 \pm 2,95$ & $31,75 \pm 16,73$ & $6,24 \pm 3,4$ & $103,8 \pm 26,57$ & $5,24 \pm 0,75$ & $4,05 \pm 0,51$ \\
\hline & 2 & $58,83 \pm 34,63$ & $14,50 \pm 8,73$ & $32,67 \pm 20,37$ & $8,66 \pm 5,36$ & $104,4 \pm 28,29$ & $2,73 \pm 2,21$ & $4,49 \pm 0,42$ \\
\hline \multirow[t]{2}{*}{ V 30} & 1 & $90,17 \pm 39,12$ & $10,80 \pm 4,17$ & $45,17 \pm 18,03$ & $6,23 \pm 4,25$ & $103 \pm 27,97$ & $5,17 \pm 0,16$ & $4,5 \pm 0,44$ \\
\hline & 2 & $74,75 \pm 21,62$ & $11,47 \pm 10,11$ & $38,0 \pm 15,67$ & $8,62 \pm 3,92$ & $111 \pm 31,55$ & $2,57 \pm 2,96$ & $4,92 \pm 0,56$ \\
\hline
\end{tabular}

FC: Freqüência cardíaca; AD: Átrio direito; PAP: Pressão de artéria pulmonar; AE: Átrio esquerdo; PAM: Pressão arterial média; DC: Débito cardíaco; Fx: Fluxo do dispositivo;V0: sem vácuo; V10: vácuo de $10 \mathrm{mmHg}$; V20: vácuo de $20 \mathrm{mmHg}$; 30 : vácuo de $30 \mathrm{mmHg}$. 1 - condição normal, 2 - após indução de insuficiência miocárdica. 
Benício A, Moreira L F P, Hayashida S, Cestari I A, Leirner A A, Stolf N A G, Jatene A D - Avaliação do desempenho hemodinâmico do dispositivo de assistência ventricular InCor como substituto do coração esquerdo. Rev Bras Cir Cardiovasc 1999; 14 (3): 237-46.

GRÁFICO 1

VALOR MÉDIO FLUXO DO DAV-INCOR COM A CANULAÇÃO ATRIAL, DE ACORDO COM O NÍVEL DO VÁCUO. SEM IC: SEM INSUFICIÊNCIA CARDÍACA; COM IC: COM INSUFICIÊNCIA CARDÍACA; VO: SEM VÁCUO; V10: VÁCUO DE 10 MMHG; V20: VÁCUO DE 20 MMHG; V30: VÁCUO DE $30 \mathrm{MMHG}$

\section{Fluxo do Dispositivo X Nível de Vácuo}

\section{$\mathrm{I} / \mathrm{min}$}

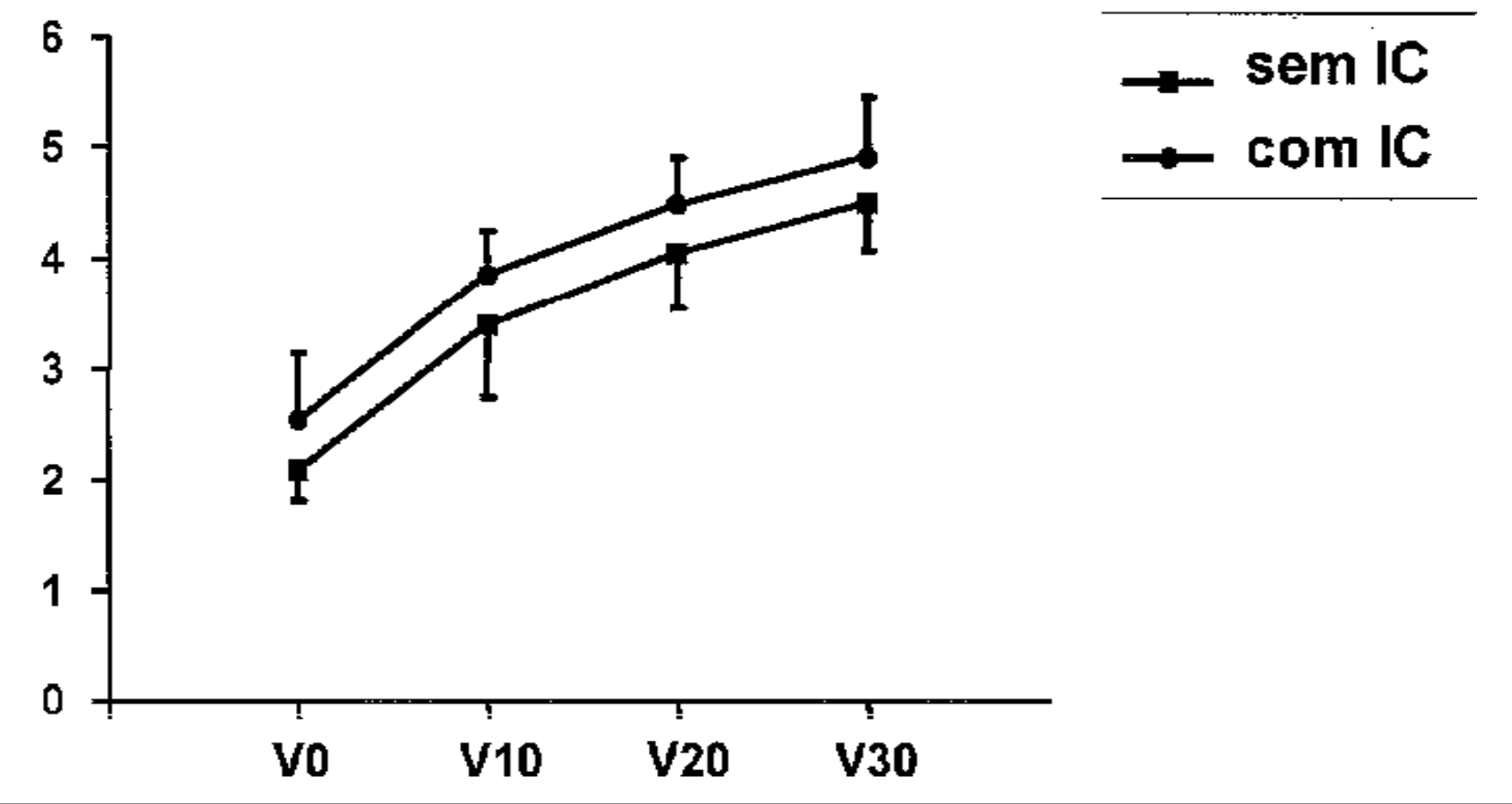

regressão polinomial. As maiores variações do desempenho do DAV-InCor com a canulação atrial foram obtidas após a introdução de $10 \mathrm{mmHg}$ de vácuo no sistema de drenagem, não havendo grande alteração do fluxo do dispositivo em relação às variações da pré-carga com níveis de vácuo acima de $20 \mathrm{mmHg}$.

\section{Grupo II}

Na Tabela 2 são apresentados os valores mé- dios e o desvio padrão da freqüência cardíaca, das pressões arterial, de átrio direito, de átrio esquerdo, de artéria pulmonar, bem como do débito cardíaco e do fluxo do DAV-InCor; obtidos com o dispositivo acionado com e sem a aplicação do vácuo de 10 $\mathrm{mmHg}$. Não houve variação significativa dos parâmetros hemodinâmicos estudados entre as duas fases do procedimento.

Com a cânula apical, o fluxo do DAV-InCor foi de 4,2 \pm 0,6 l/min na condição sem vácuo e de 4,4 \pm $0,7 \mathrm{I} / \mathrm{min}$ com vácuo de $10 \mathrm{mmHg}$, sendo observados

TABELA 2

PARÂMETROS HEMODINÂMICOS E DESEMPENHO DO DAV-InCor CÂNULA APICAL (GRUPO II)

\begin{tabular}{lccccccc}
\hline & $\begin{array}{c}\text { PA } \\
\text { (MMHG) }\end{array}$ & $\begin{array}{c}\text { FC } \\
\text { (BPM) }\end{array}$ & $\begin{array}{c}\text { AD } \\
\text { (MMHG) }\end{array}$ & $\begin{array}{c}\text { AE } \\
\text { (MMHG) }\end{array}$ & $\begin{array}{c}\text { PAP } \\
\text { (MMHG) }\end{array}$ & $\begin{array}{c}\text { DC } \\
\text { (L/MIN) }\end{array}$ & $\begin{array}{c}\text { Fx } \\
\text { (L/MIN) }\end{array}$ \\
\hline V 0 & $74,88 \pm 25,9$ & $105 \pm 18,71$ & $11,95 \pm 5,31$ & $11,10 \pm 2$ & $35,55 \pm 11,01$ & $4,44 \pm 0,98$ & $4,20 \pm 0,60$ \\
V 10 & $73,5 \pm 20,85$ & $81,50 \pm 3,5$ & $13,38 \pm 6,63$ & $10,3 \pm 3,5$ & $37,75 \pm 5,97$ & $3,46 \pm 0,99$ & $4,40 \pm 0,70$ \\
\hline
\end{tabular}

PA: Pressão arterial; FC: Freqüência cardíaca; AD: Átrio direito; AE: Átrio esquerdo; PAP: Pressão de artéria pulmonar; DC: Débito cardíaco; Fx: Fluxo do dispositivo; V0: sem vácuo; V10: vácuo de $10 \mathrm{mmHg}$. 
Benício A, Moreira L F P, Hayashida S, Cestari I A, Leirner A A, Stolf N A G, Jatene A D - Avaliação do desempenho hemodinâmico do dispositivo de assistência ventricular InCor como substituto do coração esquerdo. Rev Bras Cir Cardiovasc 1999; 14 (3): 237-46.

GRÁFICO 2

RELAÇÃO ENTRE O FLUXO DO DAV-INCOR COM A CANULAÇÃO ATRIAL E O DÉBITO CARDÍACO TOTAL, DE ACORDO COM O NÍVEL DO VÁCUO. SEM IC: SEM INSUFICIÊNCIA CARDÍACA; COM IC: COM INSUFICIÊNCIA CARDÍACA; VO: SEM VÁCUO; V10: VÁCUO DE 10 MMHG; V20: VÁCUO DE 20 MMHG; V30: VÁCUO DE 30 MMHG

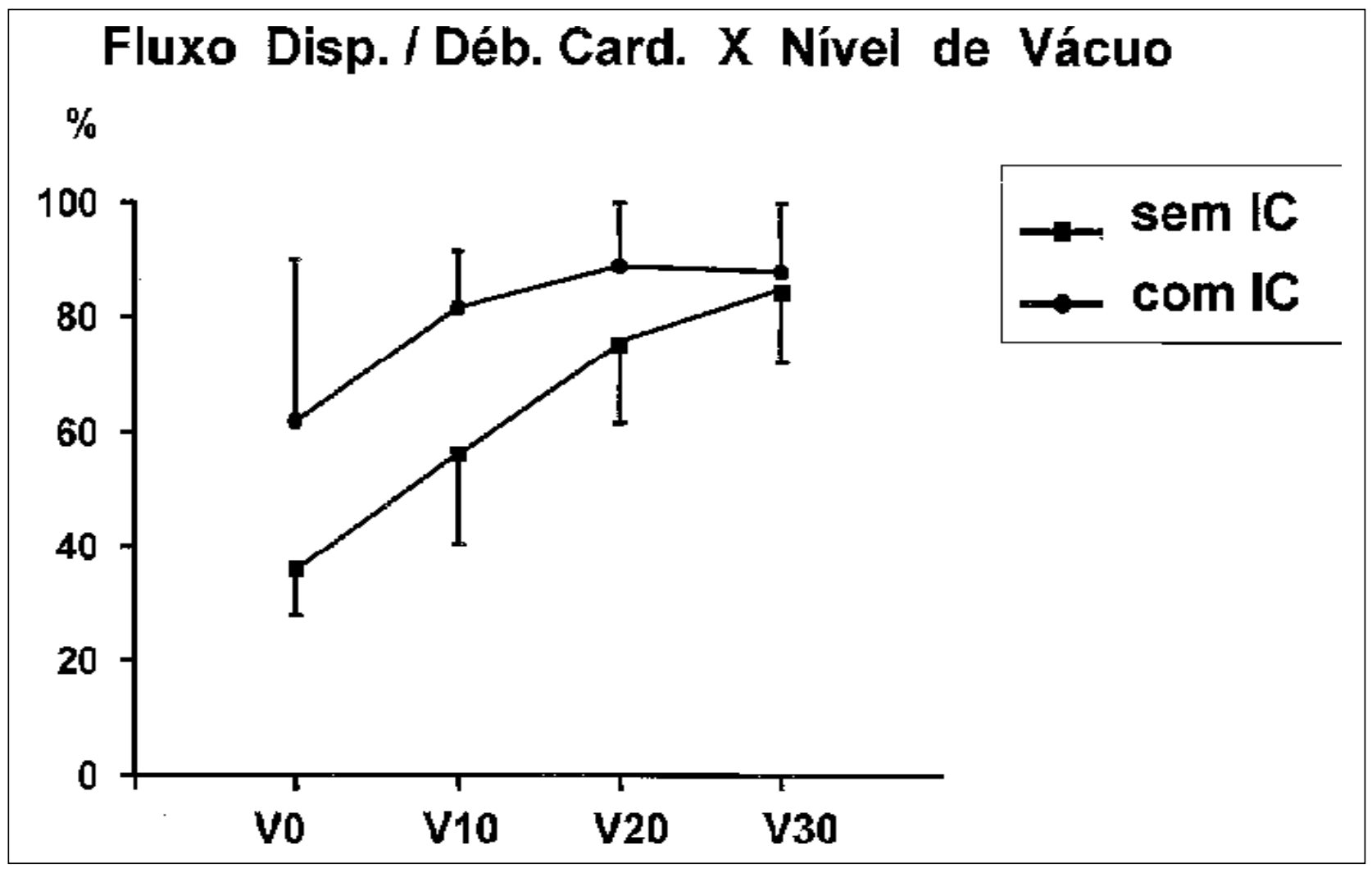

valores da pressão de átrio esquerdo de 11,1 \pm 2 e $10,3 \pm 3,5 \mathrm{mmHg}$ nas duas condições, respectivamente (Gráfico 4). Não houve diferença significativa entre os parâmetros hemodinâmicos nas duas situações estudadas. O fluxo do DAV-InCor também não mostrou alteração após a aplicação do vácuo, resultado da eficiência da drenagem apical.

Esses resultados foram observados em condições hemodinâmicas semelhantes, sendo o fluxo do DAV-InCor responsável por um porcentual maior do débito cardíaco total conforme o nível de vácuo. Esse porcentual foi de $86 \pm 13 \%$ com a cânula atrial e vácuo de $30 \mathrm{mmHg}$ e de $97 \pm 3 \%$ com a drenagem ventricular e vácuo de $10 \mathrm{mmHg}$ (Gráfico 5).

\section{COMENTÁRIOS}

O primeiro relato de uso clínico de suporte circulatório mecânico foi em 1953 com o desenvolvimento da circulação extracorpórea (7). Com o sucesso da assistência, outros dispositivos foram sendo criados com diversas formas de aplicação. Em 1967, a primeira aplicação clínica do balão intra-aórtico trouxe novas perspectivas para os pacientes com quadro de insuficiência cardíaca grave ${ }^{(8)}$.

$\mathrm{Na}$ década de 60 , existem relatos ocasionais de pacientes com insuficiência cardíaca congestiva que receberam suporte circulatório mecânico temporário, com dispositivos de assistência ventricular ou até mesmo com corações artificiais totais. Entretanto, tais procedimentos obtiveram sucesso relativo no sentido de provar que esse tipo de assistência poderia sustentar adequadamente um paciente em insuficiência cardíaca e choque cardiogênico, até que sua função cardíaca fosse recuperada ou que um coração doador pudesse ser obtido.

Paralelamente, nos anos 70 , grandes dificuldades vinham sendo encontradas no controle da imunossupressão dos pacientes transplantados, o que fez com que o transplante cardíaco não tivesse resultados satisfatórios. Por outro lado, com o advento da ciclosporina no início dos anos 80 , o transplante cardíaco tornou-se uma real perspectiva no tratamento cirúrgico da insuficiência cardíaca congestiva.

Concomitantemente ao avanço do transplante cardíaco, surgiu uma maior preocupação em rela- 
Benício A, Moreira L F P, Hayashida S, Cestari I A, Leirner A A, Stolf N A G, Jatene A D - Avaliação do desempenho hemodinâmico do dispositivo de assistência ventricular InCor como substituto do coração esquerdo. Rev Bras Cir Cardiovasc 1999; 14 (3): 237-46.

GRÁFICO 3

CURVA DE COMPORTAMENTO DO FLUXO DO DAV-INCOR COM A CANULAÇÃO ATRIAL, DE ACORDO COM A PRESSÃO DE ÁTRIO ESQUERDO

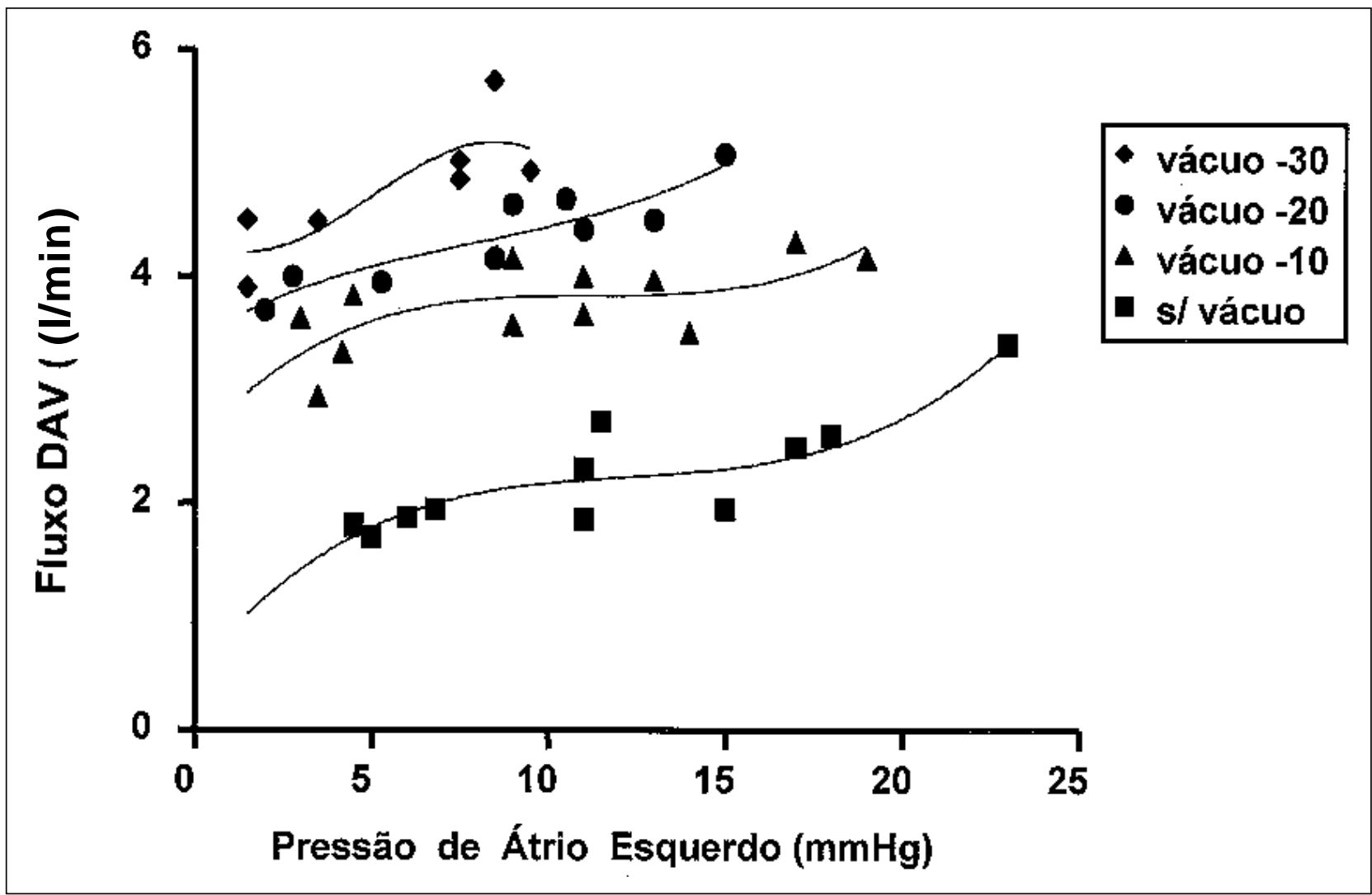

ção aos dispositivos mecânicos de assistência circulatória. Estudos clínicos se iniciaram na tentativa de estabelecer maior segurança e eficácia aos sistemas de suporte que eram empregados nos pacientes que aguardavam o transplante cardíaco. Após várias fases do desenvolvimento e aperfeiçoamento

GRÁFICO 4

VALOR MÉDIO FLUXO DO DAV-INCOR COM A CANULACÃO DO ÁPICE DO VENTRÍCULO ESQUERDO, DE ACORDO COM O NÍVEL DO VÁCUO EM MMHG

Fluxo do Dispositivo $X$ Nível de Vácuo

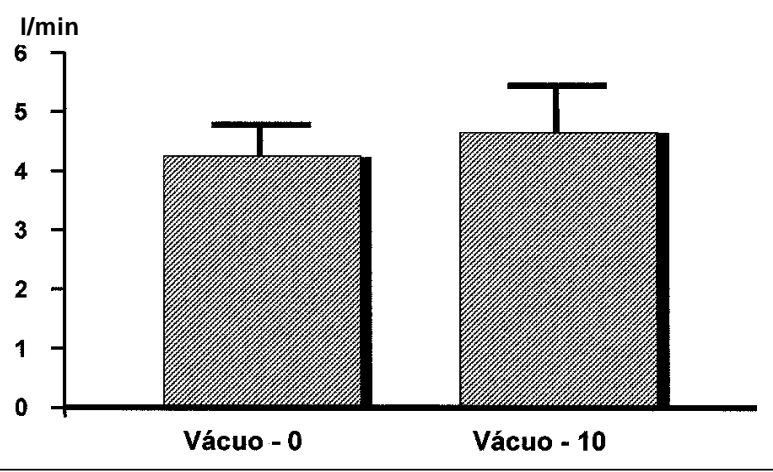

dos DAVs, eles atualmente representam métodos de suporte circulatório seguro e eficaz.

A porcentagem de pacientes que são transplantados, após o uso de suporte circulatório mecânico, varia de $62 \%$ a $69 \%$, e taxa de alta hospitalar após o transplante é de $65 \%$ a $69 \%$, independentemente

GRÁFICO 5

RELAÇÃO ENTRE O FLUXO DO DAV-INCOR COM A CANULAÇÃO DO ÁPICE DO VENTRÍCULO ESQUERDO E O DÉBITO CARDÍACO TOTAL, DE ACORDO COM O NÍVEL DO VÁCUO

Fluxo Disp. I Déb. Card. X Nível de Vácuo $\%$

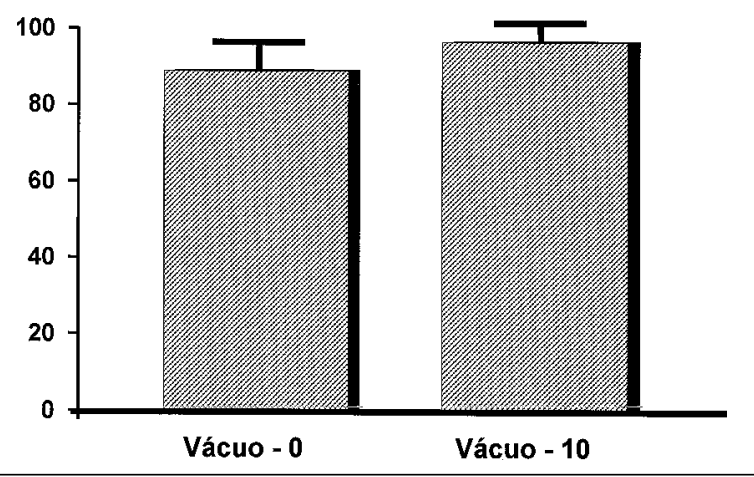


Benício A, Moreira L F P, Hayashida S, Cestari I A, Leirner A A, Stolf N A G, Jatene A D - Avaliação do desempenho hemodinâmico do dispositivo de assistência ventricular InCor como substituto do coração esquerdo. Rev Bras Cir Cardiovasc 1999; 14 (3): 237-46.

do tipo de dispositivo utilizado. Em experiências com a utilização de ventrículos artificiais pára-corpóreos ou semi-implantáveis, o transplante também tem sido realizado em aproximadamente $60 \%$ dos pacientes, com um porcentual de alta hospitalar de até $89 \%{ }^{(9)}$.

Os dispositivos de assistência circulatória mecânica representam uma valiosa opção terapêutica para o tratamento da insuficiência cardíaca refratária ao tratamento clínico. Entretanto, durante a aplicação destes dispositivos, algumas complicações surgem com determinada freqüência. O risco de sangramentos, quadros infecciosos, microembolias e disfunção ventricular direita são algumas das complicações que podem estar relacionadas ao dispositivo propriamente dito $(3,10,11)$.

Outras características podem interferir não só no quadro geral do paciente como também no desempenho do dispositivo. A aplicação ou não de vácuo no sistema de drenagem, e o posicionamento da cânula de drenagem no átrio esquerdo ou no ventrículo esquerdo são fatores que interferem diretamente no desempenho do dispositivo.

Com o modo de funcionamento do DAV-InCor "fill-to empty", a condição hemodinâmica do paciente torna-se fundamental para seu bom funcionamento, bem como uma adequada função ventricular direita. A aplicação de vácuo no sistema de drenagem promove baixos níveis pressóricos nas câmaras cardíacas esquerdas, implicando em menor consumo de oxigênio pelo miocárdio, além de fornecer maior débito para o dispositivo, tendo seu desempenho otimizado.

Entretanto, o local do implante da cânula de drenagem apresenta algumas particularidades podendo interferir diretamente no seu desempenho. Muitos estudos ainda são realizados observando o melhor posicionamento da cânula de drenagem. O quadro clínico do paciente (infarto do miocárdio, insuficiência cardíaca congestiva, ponte para transplante) pode determinar qual o tipo mais adequado de canulação. Pacientes com câmara ventricular esquerda pequena, infarto do miocárdio anterior recente (< 2 semanas) e a possibilidade de recuperação da função contrátil do ventrículo esquerdo são geralmente indicados para a canulação do átrio esquerdo (12).

Algumas desvantagens da canulação atrial esquerda são descritas: baixo fluxo do DAV quando comparado com a canulação ventricular; a descompressão do ventrículo esquerdo se faz de maneira menos completa podendo implicar em menor possibilidade de limitação da área infartada e permitir uma recuperação ventricular; e a possibilidade de embolização secundária à formação de trombo pela lentidão do fluxo no ventrículo esquerdo (12).

Contudo, o fluxo do DAV promovido pela canulação atrial mostrou-se suficiente para manter um adequado débito cardíaco neste estudo, sem que ocorresse hemólise, apesar do alto nível de vácuo aplicado.

A canulação ventricular esquerda promove não só melhor perfusão mas também melhor desempenho do DAV em relação à canulação do átrio esquerdo.

A canulação ventricular apical oferece maior fluxo do DAV e menores níveis pressóricos de átrio esquerdo e de átrio direito. Permite melhor enchimento do dispositivo, resultando em um menor tempo diastólico para o DAV, o que leva a um aumento na freqüência do DAV quando operado no sistema "fillto-empty"(13).

Os benefícios da canulação apical tornam-se evidentes quando aplicados clinicamente. Além do adequado fluxo do DAV, sendo obtido com baixos níveis pressóricos de pré-carga, resulta em menor necessidade de administração de volumes. A reduzida administração de volumes resulta em vantagens, tais como a diminuição da congestão pulmonar, extubação precoce e redução nas complicações relacionadas a politransfusões (13)

Contratempos na canulação apical residem na ocorrência de obstrução da cânula e na lesão miocárdica em pacientes recuperáveis. Na questão da preservação miocárdica, a canulação apical representa certa desvantagem. Problemas técnicos incluindo lesão muscular ventricular e dificuldade de inserção da cânula em pacientes com cavidade ventricular pequena e o trauma provocado pela subseqüente retirada da cânula são conseqüências inerentes ao procedimento. Todavia, a canulação atrial também apresenta seus contrapontos técnicos. Lesões da valva mitral e "shunt"direita-esquerda, em virtude de forma oval patente não diagnosticado, são ocorrências descritas (14).

SETHIA et al. (14) demonstraram que o efeito global da disfunção causada pela canulação apical parece ser mascarado pelo aumento compensatório na função de outra área do ventrículo esquerdo, resultando na mesma fração de ejeção global. Na presença de isquemia miocárdica ou infarto do miocárdio, os efeitos a longo prazo da canulação apical na função global do ventrículo esquerdo parecem ser mais pronunciados, com importantes implicações clínicas.

Entretanto, no caso da aplicação do DAV como ponte para transplante, quando não existe a perspectiva de recuperação da função contrátil do ventrículo esquerdo, a canulação apical acaba não resultando em dano miocárdico importante, além de criar condição para exploração e retirada de eventuais trombos da cavidade ventricular esquerda. Apesar da necessidade do estabelecimento da circulação extracorpórea para a canulação apical ( o que não ocorre na canulação atrial, exceto em casos 
Benício A, Moreira L F P, Hayashida S, Cestari I A, Leirner A A, Stolf N A G, Jatene A D - Avaliação do desempenho hemodinâmico do dispositivo de assistência ventricular InCor como substituto do coração esquerdo. Rev Bras Cir Cardiovasc $1999 ; 14$ (3): 237-46.

de importante instabilidade hemodinâmica), o DAV não necessita de níveis elevados de vácuo para ter um desempenho adequado, promovendo assim menor risco de hemólise.

\section{CONCLUSÕES}

O DAV-InCor, quando utilizado como substituto do coração esquerdo, apresentou um bom desempenho, sendo capaz de manter uma condição circulatória normal. A eficiência deste dispositivo foi melhor quando a cânula de drenagem foi inserida na porção apical do ventrículo esquerdo, sem a necessidade da aplicação de níveis elevados de vácuo no sistema de drenagem. Quando a cânula de drenagem foi inserida no átrio esquerdo o seu desempenho foi progressivamente melhor, de acordo com elevação do nível de vácuo no sistema de drenagem.

RBCCV 44205-465

Benício A, Moreira L F P, Hayashida S, Cestari I A, Leirner A A, Stolf N A G, Jatene A D - Analysis of the hemodynamic performance of the InCor ventricular assist device as a substitute for the left heart. Rev Bras Cir Cardiovasc 1999; 14 (3):237-46.

ABSTRACT: Background: The mechanical circulatory assistance is a therapeutic option in cases of cardiogenic shock refractory to the pharmacological treatment, and is frequently used as a bridge for heart transplantation.

Objective: To evaluate the action of the Ventricular Assist Device (VAD) developed by the Bioengineering Division of the Instituto do Coração, implanted as a substitute of the left heart.

Patients and Methods: Ten Girolando calves with medium weight of $73 \mathrm{~kg}$ were studied. The VAD-InCor implant was accomplished with the drainage cannula positioned in the left atrium (LA) or in the apex of the left ventricle and the replacement cannula implanted in the descending thoracic aorta. The pressures of the right and left heart, cardiac output and the flow of VAD were determined before and after the pharmacological induction of myocardial failure, at different levels of vacuum of the drainage system.

Results: Values of the flow of VAD with the drainage in LA were of $2.2 \pm 0.5 \mathrm{l} / \mathrm{min}$ without vacuum, of $3.7 \pm 0.4$ with vacuum of $10 \mathrm{mmHg}$, of $4.3 \pm 0.4$ with vacuum of $20 \mathrm{mmHg}$ and of $4.8 \pm 0.6$ with vacuum of $30 \mathrm{mmHg}$. The values of the pressure of LA were: $11.7 \pm 6 ; 9.8 \pm 5.3 ; 8.5 \pm 4.4$ and $5.6 \pm 3.3 \mathrm{mmHg}$ under the same conditions, respectively. With the ventricular cannula, the VAD flow was $4.2 \pm 0.6$ without vacuum and of $4.4 \pm 0.7$ with vacuum of $10 \mathrm{mmHg}$, with of LA pressure of $11.1 \pm 2$ and $10.3 \pm 3.5 \mathrm{mmHg}$ in the two conditions. Those results were observed in similar hemodynamic conditions, with the VAD flow responsible for a greater percentile of the total cardiac output according to the level of vacuum. That percentile was of $86 \pm 13 \%$ with the atrial cannula and vacuum of $30 \mathrm{mmHg}$ and of $97 \pm 3 \%$ with the ventricular drainage and vacuum of $10 \mathrm{mmHg}$.

Conclusions: The VAD-InCor demonstrated its effectiveness as a substitute of the left heart. The performance of this device was proportional to the level of vacuum of the drainage system and was better with the ventricular cannula.

DESCRIPTORS: Heart ventricle, surgery. Heart-assist devices.

\section{REFERÊNCIAS BIBLIOGRÁFICAS}

1 Bozkurt B \& Murali S - Ventricular assist devices as alternatives to biologic replacement of the heart. Cardiol Review 1997; 5: 243-54.

2 Pennington D G, McBride L R, Peigh P S, Miller L W, Swartz M T - Eight years' experience with bridging to cardiac transplantation. J Thorac Cardiovasc Surg 1994; 107: 472-81.

3 Massad M G \& McCarthy P M - Will permanent LVADs be better than heart transplantation? Eur J Cardiothorac Surg 1997; 11 (Suppl): S11-7.
4 Oshiro M S, Hayashida S A, Maizato M J et al. - Design, manufacturing, and testing of a paracorporeal pulsatile ventricular assist device: São Paulo Heart Institute VAD. Artif Organs 1995; 19: 274-9.

5 Bocchi E A, Vieira M L, Fiorelli A et al. - Perfil hemodinâmico e neuro-humoral durante assistência circulatória com ventrículo artificial heterotópico seguida de transplante cardíaco. Arq Bras Cardiol 1994; 62: 23-7.

6 Cestari LA, Hayashida A S, Moreira L F P et al. Avaliação do desempenho in vivo do dispositivo de assistência ventricular (DAV) InCor. In: Anais do IV 
Benício A, Moreira L F P, Hayashida S, Cestari I A, Leirner A A, Stolf N A G, Jatene A D - Avaliação do desempenho hemodinâmico do dispositivo de assistência ventricular InCor como substituto do coração esquerdo. $\quad$ Rev Bras Cir Cardiovasc 1999; 14 (3): 237-46.

F N C T S - Fórum Nacional de Ciência e Tecnologia em Saúde, 1998: 395-6.

7 Hunt S A \& Frazier $\mathrm{OH}$ - Mechanical circulatory support and cardiac transplantation. Circulation 1998; 97: 2079-90.

8 Kantrowitz A, Wasfie T, Freed P S, Rubenfire M, Wajszczuk W, Schork M A - Intraaortic balloon pumping 1967 through 1982: analysis of complications in 733 patients. Am J Cardiol 1986; 57: 976-83.

9 Arabia F A, Smith R G, Rose D S, Arzouman D A, Sethi G K, Copeland J G - Success rates of longterm circulatory assist devices used currently for bridge to heart transplantation. ASAIO J 1996; 42: M542-6.

10 Roberts J K, Mohr J P, Moazami N, Oz M C - Micro- embolic signals in patients with left ventricular assist devices. Stroke 1996; 27: 1915-6.

11 Elbeery J R, Owen C H, Savitt M A et al. - Effects of the left ventricular assist device on right ventricular function. J Thorac Cardiovasc Surg 1990; 99: 809-16.

12 Holman W L, Bourge R C, Murrah C P et al. - Left atrial or ventricular cannulation beyond 30 days for a Thoratec ventricular assist device. ASAIO J 1995; 41: M517-22.

13 Lohmann D P, Swartz M T, Pennington D G, McBride L R, Reedy J E, Miller $L$ - Left ventricular versus left atrial cannulation for the Thoratec ventricular assist device. ASAIO Trans 1990; 36: M545-8.

14 Sethia B, Martin W, Wheatley D J - The effects of left atrial and left ventricular cannulation on left ventricular function. Int J Artif Organs 1985; 8: 331-4. 